\title{
COMENTARIO EDITORIAL
}

Perspectivas sobre el uso de Estatinas en pacientes con colesterol normal

En mayo de 1998 se publicó el primer ensayo clínico randomizado y controlado que demostró la eficacia de las estatinas en la prevención primaria de eventos coronarios en pacientes con niveles de colesterol total considerados "normales" (CT $221+/-21 \mathrm{mg} \%$, LDL $150+/-17 \mathrm{mg} \%$ ) y niveles levemente disminuídos de $\mathrm{HDL}-\mathrm{C}(36+/-5 \mathrm{mg} \%$ en hombres y $40+/-5 \mathrm{mg} \%$ en mujeres $)^{1}$. En el estudio AFCAPS / TEXCAPS, a lo largo de poco más de 5 años de seguimiento, la incidencia de un primer evento coronario mayor (infarto fatal - no fatal, angina inestable o muerte súbita) se redujo un $37 \%$ [IC 95\% 21\% - 50\%] en aquellos pacientes tratados con lovastatina 20 a $40 \mathrm{mg} /$ día comparado con placebo. Otros eventos coronarios individualmente evaluados también demostraron una reducción significativa. Globalmente, la lovastatina redujo el LDL-C un 25\% y aumentó el HDL-C un 6\%. Ambos grupos estaban bien balanceados con respecto a variables demográficas y otros factores de riesgo coronario como hipertensión arterial, tabaquismo, diabetes, historia familiar, uso de medicación incluyendo aspirina, etc.

Es interesante destacar que este estudio confirma un patrón consistente de efectos beneficiosos de este grupo de drogas en poblaciones con muy diferentes riesgos de cardiopatía isquémica $(\mathrm{CI})^{2}$. El estudio $4 S^{3}$ de prevención secundaria en pacientes con hipercolesterolemia, incluyó el grupo de mayor riesgo; el CARE 4 , también de prevención secundaria, incluyó pacientes con niveles normales de colesterol y el WOSCOPS 5 demostró la eficacia del pravastatina en prevención primaria en pacientes sin CI pero con riesgo elevado de sufrir eventos coronarios. El AFCAPS extiende estos beneficios a individuos sin CI y con niveles considerados "normales" de colesterol total y LDL colesterol. Considerando la serie de estudios que sugieren un papel importante de las estatinas en la alteración de mecanismos trombóticos e inflamatorios de la pared arterial (que pueden contribuir a explicar el éxito de estas drogas en la reducción de eventos vasculares independientemente del descenso del colesterol), la evidencia actual demuestra sin lugar a dudas la eficacia de estas drogas en la prevención primaria y secundaria de los eventos coronarios. La editorial que acompaña al artículo de Pearson y col 2 plantea que, aún pacientes con muy bajo riesgo tratados durante un tiempo suficientemente prolongado obtendrían beneficios en cuanto a la reducción de los eventos.

Entonces: basándonos en su probada eficacia ¿deberíamos ofrecer el tratamiento con estatinas a todos?.

Obviamente, la pregunta así planteada presenta aristas muy filosas, ya que la respuesta será probablemente distinta dependiendo del punto de vista que uno adopte. Planteado desde otro lugar: ¿cómo decidimos quiénes no deberían re- cibir un tratamiento preventivo eficaz de la enfermedad coronaria?. Los estudios de prevención secundaria de la enfermedad coronaria han mostrado su costo-efectividad dado el elevado riesgo absoluto de eventos en esa población. Esto no es tan simple en prevención primaria donde la tasa de eventos es mucho menor y el tratamiento propuesto es costoso para los pacientes y los sistemas de salud. Los autores indican que tratando 1000 pacientes durante 5 años se reducen 12 IAM, 7 anginas inestables, 17 revascularizaciones coronarias y 12 eventos fatales a un costo aproximado de drogas de 1.4 millones de do lares al año (similar a nuestro país) sin contar con los costos agregados de las visitas al médico y los tests de laboratorio. En general, los estudios de costoefectividad reportan como resultado a evaluar el costo por año de vida salvado. Se estima que una intervención que genere un costo no mayor a $\$ 50.000 /$ año de vida salvado se considerará costo-efectiva. En prevención primaria es muy difícil demostrar diferencias en eventos tales como mortalidad y, por lo tanto, los estándares habituales de los estudios de costo-efectividad no se pueden aplicar fácilmente. De todas maneras, con los costos actuales, los resultados del AFCAPS/TEXCAPS demuestran un alto costo por evento prevenido.

El uso óptimo de las estatinas dependerá de tres factores $^{6}:$ 1) mejor definición de los beneficios, costos y riesgos del tratamiento basada en ensayos randomizados, 2) disminución del costo del tratamiento y 3) métodos para estimar los riesgos en los pacientes individuales.

Si bien el AFCAPS/TEXCAPS es un buen punto de partida al demostrar eficacia del tratamiento en un grupo de bajo riesgo, también demuestra la necesidad de considerar otros factores antes de recomendar su uso indiscriminado en la población general.

Es muy importante establecer criterios clínicos válidos para evaluar y estratificar el riesgo individual de cardiopatía isquémica en nuestros pacientes y así facilitar la decisión sobre el tratamiento. No existen dudas que el riesgo es alto en aquellos con enfermedad coronaria o vascular clínicamente evidente y es este el grupo en el que el tratamiento va a producir mayores beneficios. Las dificultades se presentan cuando debemos decidir el inicio del tratamiento en pacientes con riesgo bajo, hasta hoy sanos. Cuanto mejor logremos predecir subgrupos de riesgo, más eficiente será nuestra intervención. Para lograr mayor precisión deben tomarse en consideración todos los factores de riesgo coronario y no solo los niveles de colesterol. En general se recomienda el cociente Colesterol Total/ HDL-C ya que es más estable que el colesterol total y el cambio de este cociente se correlaciona bien con la disminución del riesgo. El Comité de 
Guías para el uso de estatinas de Gran bretaña (Standing Medical Advisory Committee) distribuyó recientemente las recomendaciones a los médicos ${ }^{7}$ y generó un acalorado debate. Estas guías estan basadas en la tabla de Sheffield 8 que evalúa riesgos tomando en cuenta no solo colesterol sino también edad, sexo, presión arterial, tabaquismo, diabetes e hipertrofia ventricular izquierda en el ECG y recomienda iniciar tratamiento con estatinas en aquellos pacientes que tienen un riesgo de eventos igual o mayor al 3\% anual. Esta guía ha sido criticada por presentar un punto de vista muy conservador y no ser adecuada para medir riesgos en prevención primaria. Por otro lado, la guía basada en la tabla de Nueva Zelandia ${ }^{9}$ evalúa el riesgo absoluto de sufrir un evento cardiovascular tomando en cuenta la presencia de los otros factores de riesgo. Presenta una tabla con códigos de colores según el riesgo y las recomendaciones pertinentes. Si bien esta guía también recomienda iniciar tratamiento con drogas hipolipemiantes si el riesgo anual de eventos es igual o mayor al 3\%, permite considerar niveles alternativos de riesgo para iniciar intervenciones (http//www.nzgg.org.nz/).

El precio de las estatinas es, en todo el mundo, la variable determinante del costo del tratamiento. Por lo tanto, con un presupuesto fijo y limitado, el precio de la droga afecta directamente nuestra capacidad para prevenir eventos coronarios. Los médicos somos responsables de un gran componente del gasto en salud y éste es muchas veces generado por una incorrecta interpretación de la información que nos lleva a indicar estudios complementarios o tratamientos innecesarios o inadecuados. Los recursos de cualquier sistema de salud son finitos y limitados y lo que se gasta por un lado es dinero que no se tendrá para invertir en otro.

Muchos de los estudios de eficacia y de costo-efectividad de las estatinas son investigaciones financiadas directamente por la industria farmacéutica. Estos son coordinados y llevados a cabo por renombrados investigadores y, si bien la evidencia es incontrovertible, no se puede ignorar el rédito que tienen las compañías en ampliar potencialmente el mercado de estas drogas. En el contexto actual de progresiva disminución de financiación independiente para la investigación clínica, la dirección que se le imprime a la generación de información esta más y más relacionada a la presentación de nuevas intervenciones terapéuticas o a la utilización de aquellas ya establecidas en nuevos escenarios. Dependerá en gran parte de nosotros la interpretación y aplicación responsable de estas evidencias.

\section{Dr. Fernando Rubinstein}

Unidad de Medicina Familiar y Preventiva. Hospital Italiano de Buenos Aires

1. Downs JR, Clearfield M, Weis S. Primary prevention of acute coronary events with lovastatin in men and women with average cholesterol levels: results of the AFCAPS/TexCAPS. JAMA, 1998;279:1615-1622

2. Pearson T. Lipid lowering therapy in low risk patients. JAMA,1998;279:1659-1661

3. Scandinavian Simvastatin survival Study. Lancet 1994;344:1383-1389

4. Sacks FM, Pfeffer MA, Moye LA. The effect of pravastatin on coronary events after myocardial infarction in patients with average cholesterol levels. $\mathrm{N}$ Engl $\mathrm{J}$ med. 1996;335:1001-1009.

5. Shepherd J, Cobbe S, Ford I, for the west of scotland coronary prevention study group. Prevention of coronary heart disease with pravastatin in men with hypercholesterolemia. N Engl J med. 1995;333: 1301-1307.

6. Muldoon M. Criqui M. The emerging role of statins in the prevention of coronary heart disease. BMJ 1997;315:1554-1555.

7. Standing Medical Advisory Committee. The use of statins. London:1997.

8. Ramsay L, Haq I, Jackson P. The sheffield table for primary prevention of coronary heart disease: corrected. Lancet 1996;348:1251-1252.

9. National Heart Foundation. Clinical guidelines for the assessment and management of dyslipidemia. NZ Med J 1996; 109:224-232
} 